\title{
Steady state imaging of the thoracic vasculature using inversion recovery FLASH and SSFP with a blood pool contrast agent
}

\author{
Mauricio S Galizia ${ }^{1,2^{*}}$, Jennifer A Febbo ${ }^{1}$, Andrada R Popescu ${ }^{1,3}$, Xiaoming Bi ${ }^{4}$, Jeremy Collins ${ }^{1}$, Michael Markl ${ }^{1}$, \\ Robert R Edelman², James Carr ${ }^{1}$
}

From 15th Annual SCMR Scientific Sessions

Orlando, FL, USA. 2-5 February 2012

\section{Background}

Although contrast-enhanced first-pass MRA (FP-MRA) is frequently used to visualize the thoracic vasculature, it may not be ideal for the assessment of the aortic root due to poor image quality and motion artifact. Bloodpool contrast agents remain within the intravascular space for several hours, allowing vessels to be imaged longer and therefore improving spatial resolution. The purpose of this study is to compare steady-state magnetic resonance angiography (SS-MRA) following injection of a blood-pool contras agent to first-pass MR angiography (FP-MRA) in adults with thoracic aortic disease.

\section{Methods}

25 patients (14 men, 11 women) with suspect thoracic aortic disease disease underwent MRA on a $1.5 \mathrm{~T}$ scanner (Magnetom Aera and Avanto; Siemens Medical Solutions). The MRA protocol consisted of FP-MRA followed by SS-MRA after intravenous injection of gadofosveset trisodium (Ablavar, Lantheus Medical Imaging). FP-MRA consisted of a breath-held ECG-gated FLASH acquisition in a sagittal oblique orientation with the following imaging parameters: TR/TE: 2.8/1.0, flip angle $25^{\circ}$, FOV $343 \times 500 \mathrm{~mm}$, matrix $264 \times 512$, slice thickness $1.5 \mathrm{~mm}$, voxel size $1.3 \times 1.0 \times 1.0 \mathrm{~mm}$, GRAPPA $\times 2,20$ second acquisition. $0.03 \mathrm{mmol} / \mathrm{kg}$ of gadofosveset was injected intravenously at $1 \mathrm{cc} / \mathrm{sec}$ in an antecubital vein. Contrast bolus timing was achieved using care bolus technique. SS-MRA consisted of free-breathing ECGgated IR-FLASH and IR-SSFP in a sagittal oblique

${ }^{1}$ Radiology, Northwestern University, Chicago, IL, USA

Full list of author information is available at the end of the article orientation. IR-FLASH had the following parameters: TR/TE/TI: $3.5 / 1.5 / 260$, flip angle $18^{\circ}$, and IR-SSFP had: TR/TE/TI: $3.3 / 1.5 / 260$, flip angle $70^{\circ}$. Both sequences had FOV 326x380, matrix 440x512, slice thickness 1.5 $\mathrm{mm}$, voxel $0.7 \times 0.7 \times 1.0 \mathrm{~mm}$, GRAPPA $\times 2$, and $3 \mathrm{~min}-$ ute acquisition. Respiratory gating was achieved using a navigator acquisition with an average acceptance window of $35 \%$. For quantitative analysis, orthogonal dimensions of the thoracic aorta were measured at several locations. Signal-to-noise ratio (SNR) was also measured for both techniques by placing regions of interest in the aortic root and the ascending aorta. For qualitative analysis, two independent reviewers evaluated both FP-MRA and SS-MRA images separately. The aortic root and the ascending aorta were scored on an image quality scale of 1-4.

\section{Results}

There was no significant difference in aortic dimensions at all anatomic locations between FP-MRA and SSMRA. SNR was higher for SS-MRA compared to FPMRA. Image quality scores were higher for SS-MRA compared to FP-MRA. Thoracic aortic aneurysms were detected equally between both techniques.

\section{Conclusions}

SS-MRA following injection of blood pool contrast agent was comparable to FP-MRA for measurement of aortic dimensions. SNR and image quality was higher with SS-MRA. SS-MRA may be a useful adjunct to FPMRA in cases of bolus mistiming, or may potentially replace FP-MRA, thereby simplifying MRA assessment of the thoracic vasculature. 


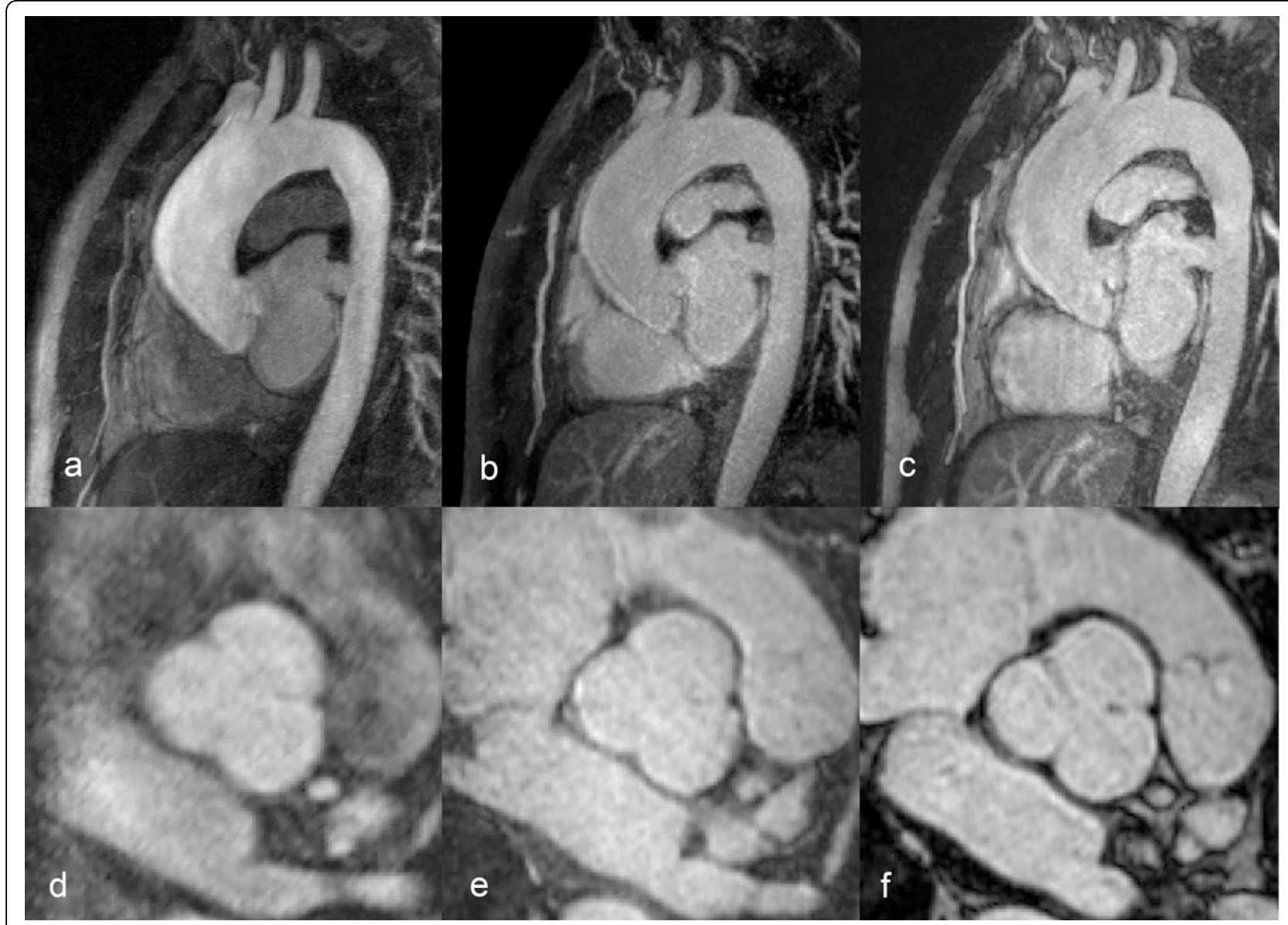

Figure 1 MRA of the thoracic aorta of a 57-year-old man in a sagittal oblique orientation (a-c) and in a multiplane reformat image (MPR) orthogonal to the sinus of Valsalva (d-f). The image quality of FP-MRA $(a, d)$ is not as high as in the SS-MRA images: IR-FLASH (b, e) and IR-SSFP $(c, f)$.

\section{Funding}

None.

\section{Author details}

${ }^{1}$ Radiology, Northwestern University, Chicago, IL, USA. ${ }^{2}$ Radiology,

NorthShore University HealthSystem, Evanston, IL, USA. ${ }^{3}$ Medical Imaging,

Children's Memorial Hospital, Chicago, IL, USA. ${ }^{4}$ Cardiovascular MR R\&D,

Siemens Healthcare, Chicago, IL, USA.

Published: 1 February 2012

\section{doi:10.1186/1532-429X-14-S1-P51}

Cite this article as: Galizia et al: Steady state imaging of the thoracic vasculature using inversion recovery FLASH and SSFP with a blood pool contrast agent. Journal of Cardiovascular Magnetic Resonance 201214 (Suppl 1):P51.

\section{Submit your next manuscript to BioMed Central and take full advantage of:}

- Convenient online submission

- Thorough peer review

- No space constraints or color figure charges

- Immediate publication on acceptance

- Inclusion in PubMed, CAS, Scopus and Google Scholar

- Research which is freely available for redistribution 